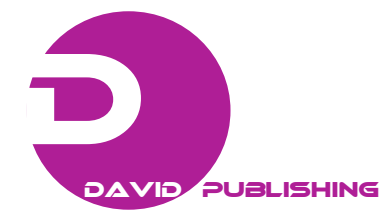

\title{
Prediction for Growth of Staphylococcus aureus Affected by Storage Temperature in Sausage
}

Bo-Geum Park, Myoung-Su Park, Xihong Zhao, Jun Wang, Joong-Hyun Park, Fereidoun Forghani, Gwang-Hee Kim, Deog-Hwan Oh*

Department of Food Science and Biotechnology and Institute of Bioscience and Biotechnology, Kangwon National University,

Chuncheon, Gangwon 200-701, Republic of Korea

*Corresponding author's E-mail: deoghwa@kangwon.ac.kr

\begin{abstract}
Sausage, which is popular processed meat product, is widely consumed in many countries. The objective of the present study was to investigate the growth of Staphylococcus aureus influenced by storage temperature in sausage and develop predictive models. The experimental data at each temperatures $\left(15,20,25,30\right.$ and $\left.35{ }^{\circ} \mathrm{C}\right)$ were fitted into the primary models. The modified Gompertz model was estimated the best primary model. The growth parameters such as growth rate (GR) and lag time (LT) calculated from the modified Gompertz model with high determination of coefficients $\left(\mathrm{R}^{2}\right)$ were employed to develop the square root model as secondary model. In order to verify that the developed model offers dependable prediction for the growth of $S$. aureus in accordance with storage temperature, bias factor $\left(B_{f}\right)$, accuracy factor $\left(A_{f}\right)$, and root mean square error (RMSE) were estimated. $B_{f}$, $A_{f}$ and RMSE were within the reliable rage. The results described that the established models have enough ability to predict the microbial growth of S.aureusin sausage and can be used for risk assessment.
\end{abstract}

Key words: Staphylococcus aureus, sausage, predictive model, temperature, evaluation. 